\title{
TTR
}

Traduction, terminologie, re?daction

\section{Wolfram Wilss. Translation and Interpreting in the 20th century: Focus on German. Amsterdam/Philadelphia, John Benjamins, 1999, 258 p.}

\section{Hannelore Lee-Jahnke}

Volume 12, numéro 2, 2e semestre 1999

Poésie, cognition, traduction II - Autour d'un poème de W. H. Auden

Poetry, Cognition, Translation II — On a Poem by W. H. Auden

URI : https://id.erudit.org/iderudit/037385ar

DOI : https://doi.org/10.7202/037385ar

Aller au sommaire du numéro

Éditeur(s)

Association canadienne de traductologie

ISSN

0835-8443 (imprimé)

1708-2188 (numérique)

Découvrir la revue

Citer ce compte rendu

Lee-Jahnke, H. (1999). Compte rendu de [Wolfram Wilss. Translation and Interpreting in the 20th century: Focus on German. Amsterdam/Philadelphia, John Benjamins, 1999, 258 p.] TTR, 12(2), 202-205.

https://doi.org/10.7202/037385ar d'utilisation que vous pouvez consulter en ligne.

https://apropos.erudit.org/fr/usagers/politique-dutilisation/ 
Wolfram Wilss. Translation and Interpreting in the 20th century : Focus on German. Amsterdam/Philadelphia, John Benjamins, 1999, $258 \mathrm{p}$.

Une fois de plus, Wolfram Wilss comble une lacune en traductologie: l'histoire de la traduction et de l'interprétation en Allemagne de 1900 à nos jours.

Dans sa préface, l'auteur précise bien qu'il s'agit de donner aux lecteurs un aperçu du développement de la médiation langagière $\mathrm{du}$ $\mathrm{XX}^{\mathrm{e}}$ siècle. Et ceci en Allemagne. Le livre est structuré de telle façon que le lecteur pressé peut faire un choix de lectures sans être tenu de lire l'ouvrage du début à la fin. Le fait que l'auteur sait marier la théorie à la pratique ressort dès la lecture de la table des matières, car les deux volets reçoivent un traitement égal. Comme presque toujours, Wilss traite toute problématique de façon aussi neutre que possible : il justifie ses prises de position, qu'elles soient positives ou négatives. Wilss est en outre très soucieux de ses lecteurs : il leur fournit une bibliographie des plus récentes ainsi qu'un index par nom d'auteurs et par sujets.

Le chapitre 1, Rétrospective, contient, entre autres, un certain nombre d'anecdotes de l'histoire de la traduction avant l'ère qui nous interesse ici. Le lecteur voit ainsi défiler devant ses yeux les noms de ceux qui ont fait l'histoire de la traduction, cette histoire qui s'écrit avec un grand " $\mathrm{H}$ ", tels que Cicéron, saint Jérôme et Luther. Ce même chapitre fournit également l'explication de certaines notions fondamentales. C'est ainsi que l'acte de traduire est subdivisé en trois catégories : a) la traduction de textes bibliques; b) la traduction de textes littéraires; c) la traduction de textes spécialisés. Subdivision tripartite également pour l'interprétation.

Une fois cela établi, les chapitres 2,3 et 4 traitent de périodes bien définies : 1900-1919; 1919-1945; 1945-1990, respectivement. Le sous-chapitre 2.1 qui est intitulé The Auswärtige Amt in Bonn (L'Office des affaires étrangères), nous apprend que jusqu'en 1918, le mot dragoman servait à désigner les interprètes pour les langues exotiques. La formation de ces dragomans nous est décrite en détail et 
les exigences peuvent étonner : à titre d'exemple, le diplôme universitaire en droit était un des préalables.

Au chapitre 3, Wilss relève l'importance de la distinction qui sera désormais faite entre traduction et interprétation et indique en détail les débuts de l'institutionnalisation de la formation de ces médiateurs linguistiques. Tout formateur de traducteur dont la curiosité aura été piquée par cet intitulé remarquera à la lecture, que certains problèmes de jadis continuent, hélas, à persister.

Le chapitre 4 décrit l'époque de la Deuxième Guerre mondiale : structures et mécanismes toujours plus complexes au niveau de la communication internationale. Et, comme un des sous-chapitres l'indique, le bilinguisme fait place au multilinguisme, le meilleur exemple étant l'Union Européenne avec ses onze langues officielles.

Le livre comporte ici, à notre avis, une césure : après avoir décrit la situation des langagiers et des professionnels de la traduction et de l'interprétation, l'auteur présente au chapitre 5 les différents domaines de spécialisation. C'est ici qu'est donnée toute l'importance à la terminologie et aux banques de données telles qu'EURODICOTOM et TERMDAT.

Le chapitre 6, le plus volumineux, traite de quelques exemples pertinents de la pratique afin de mieux comprendre la situation actuelle. Wilss applique souvent cette procédure, nous l'avons vu au début du livre. Les aspects à la fois professionnels et pédagogiques se trouvent à l'avant-plan. On ne pouvait pas manquer, en ce qui concerne l'Allemagne, de parler de The Academicization of Training (6.8), qui est probablement la raison profonde de toutes les divergences autour de l'épineux problème de la théorie vs la pratique. Ce n'est pas la première fois que l'auteur aborde cette question. (Nous faisons allusion ici à son ouvrage Übersetzungsunterricht, Eine Einführung, publié en 1996). Les deux derniers sous-chapitres (6.12 et 6.13) traitent du statut $d u$ traducteur, de la reconnaissance sociale, qui est faible en Allemagne. Wilss donne son explication pour cet état de choses :

The feeling of uncertainty that most translators have is due to the fact that they work in isolation, carrying out a specific task entirely under their own steam. (1999, p. 177) 
La reconnaissance, ou non-reconnaissance devrait-on dire, se remarque également dans la position qu'occupent les différents instituts de formation de traducteurs et interprètes au sein des universités et dans les difficultés liées à une reconnaissance académique de la profession. Ces deux sous-chapitres, qui traitent d'un aspect très important, auraient pu faire l'objet d'un chapitre séparé, à notre avis.

Les chapitres 7 et 8 donnent un survol de la situation sur le marché du travail et présentent, de ce fait, un intérêt tout particulier pour l'apprenant. L'auteur y fait mention, entre autres, d'une étude qu'il a effectuée au début des années 70 (la situation a-t-elle évolué depuis 30 ans?) pour examiner de près l'offre et la demande au niveau de la traduction. Nous apprenons que de nombreuses entreprises préfèrent investir dans les connaissances linguistiques de leurs employés, plutôt que d'employer des traducteurs formés. Wilss en tire la conclusion que les concepts de formation doivent être adaptés en offrant, par exemple, des études de niveau supérieur de spécialisation. L'informatisation et les nouvelles technologies deviennent une conditio sine qua non pour le traducteur qui veut s'affirmer sur le marché. Wilss clôt son ouvrage avec la constatation suivante :

What is required today are practical know-how, mental agility and a balance of rationality and imagination, routine and creativity. Acquiring such "key qualifications" in a carefully planned and methodical training course seems to me to be the most important thing in improving translator competence or, as one might also put it, "translator maturity". (1999, p. 236)

Tout semble donc faisable. Mais le chemin n'est certes pas des plus faciles!

Le livre de Wilss se lit facilement et on relève, surtout dans la première partie, un ton légèrement ironique. Le style est parfois anecdotique et les exemples qu'il choisit pour étayer ses arguments sont souvent amusants. C'est un vrai plaisir! Pour un public étudiant non-germanophone, il aurait été utile d'ajouter quelques explications pour décrire certaines réalités propres à l'Allemagne. Tâche qui peut cependant être assumée par un enseignant.

Disons, pour conclure, que tout lecteur trouvera un intérêt à lire cet ouvrage qui s'adresse à un public très large et non défini : le professeur, le praticien, l'étudiant et, bien entendu, toute personne 
intéressée par l'évolution de la traduction et de l'interprétation en Allemagne pendant la période examinée, c'est-à-dire tout le $\mathrm{XX}^{\mathrm{e}}$ siècle.

Hannelore Lee-Jahnke Université de Genève 